\title{
Mechanical Stress to Cell Nucleus Inhibits Proliferation and Differentiation of Vascular Smooth Muscle Cells
}

\author{
Kazuaki Nagayama \\ Micro-Nano Biomechanics Laboratory, Department of Mechanical Systems Engineering, Ibaraki University, Ibaraki, Japan \\ Email: kazuaki.nagayama.bio@vc.ibaraki.ac.jp
}

How to cite this paper: Nagayama, K. (2020) Mechanical Stress to Cell Nucleus Inhibits Proliferation and Differentiation of Vascular Smooth Muscle Cells. Journal of Biosciences and Medicines, 8, 132-141. https://doi.org/10.4236/jbm.2020.85013

Received: March 9, 2020

Accepted: May 6, 2020

Published: May 9, 2020

Copyright $\odot 2020$ by author(s) and Scientific Research Publishing Inc. This work is licensed under the Creative Commons Attribution International License (CC BY 4.0).

http://creativecommons.org/licenses/by/4.0/

\section{(c) (i) Open Access}

\begin{abstract}
Cells sense the external environment such as a surface topography and change many cellular functions. Cell nucleus has been proposed to act as a cellular mechanosensor, and the changes in nuclear shape possibly affect the functional regulation of cells. This study demonstrated a large-scale mechanical deformation of the intracellular nucleus using polydimethylsiloxane (PDMS)-based micropillar substrates and investigated the effects of nuclear deformation on migration, proliferation, and differentiation of vascular smooth muscle cells (VSMCs). VSMCs spread completely between the fibronectin-coated pillars, leading to strong deformations of their nuclei resulted in a significant inhibition of the cell migration. The proliferation and smooth muscle differentiation of VSMCs with deformed nuclei were dramatically inhibited on the micropillars. These results indicate that the inhibition of proliferation and VSMC differentiation resulted from deformation of the nucleus with high internal stress, and this type of large-scale nuclear mechanical stress might lead the cells to a "quiescent state".
\end{abstract}

\section{Keywords}

Cell Biomechanics, Mechanobiology, Nuclear Mechanotransduction, Microfabrication

\section{Introduction}

Cells sense the external environment and translate this information into biochemical signals that induce various cell responses. The transport of macromolecules between the nucleus and cytoplasm is believed to have a central role in these intracellular signal transduction processes [1]; nuclear transport of proteins and RNA through the nuclear pore complex is mediated by the biochemi- 
cal activation of transport receptors, and the machinery that mediates nucleocytoplasmic exchange directly affects gene expression in cells [2].

Recent studies have suggested that biomechanical cues are also important factors for directing cell events, such as cell proliferation [3], differentiation [4], apoptosis [5], and gene expression [6]. The nucleus is the largest and stiffest organelle in cells [7] and is exposed to the mechanical forces transmitted through the cytoskeleton from outside the cell [8] [9]. The nucleus itself has been proposed to act as a cellular mechanosensor, and the changes in nuclear shape or volume induced by the area controlling cell adhesion possibly affect the regulation of cell proliferation [10] [11]. However, the investigation of nuclear mechanosensing is still very recent and numerous questions regarding the physiological roles of the nuclear deformation remain unclear.

A present study investigated the effects of nuclear deformation on cellular events, such as cell migration, proliferation, and differentiation using microfabricated cell culture substrates with an array of micropillars. Vascular smooth muscle cells (VSMCs) were used to determine the effects of the micropillar-induced nuclear deformation on normal healthy cells. The migration, proliferation, and contractile differentiation of VSMCs were measured on the micropillar substrates and the effects of a large scale of nuclear deformation with the micropillar substrates on cellular functions were discussed.

\section{Materials and Methods}

\subsection{Substrate Preparation}

The micropillar array substrates were prepared as described previously [12]. Briefly, an array of photoresist posts was made on a silicon wafer by standard photolithography as a male mold. To make a female mold for the array of microposts, poly(dimethylsiloxane) (PDMS; Sylgard 184, Dow-Corning, Midland, MI, USA) was poured over the male mold, cured at $70^{\circ} \mathrm{C}$ for $6 \mathrm{~h}$, peeled off, and silanized to aid the release of PDMS from the mold. PDMS was then poured into the female mold, cured at $100^{\circ} \mathrm{C}$ for $3 \mathrm{~h}$, and peeled off to obtain the micropillar array substrate. In this study, we designed the PDMS micropillar array substrates with a hexagonal arrangement and with a pillar diameter, length, and center-to-center spacing of 3, 9, and $9 \mu \mathrm{m}$, respectively (Figure 1 ). The Young's modulus of the

(A)

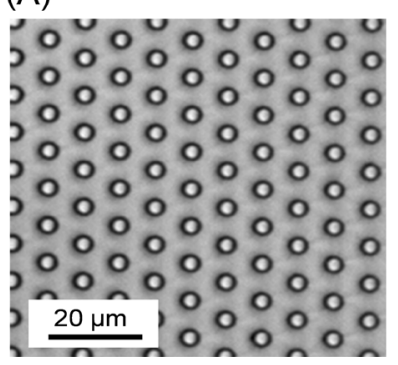

(B)

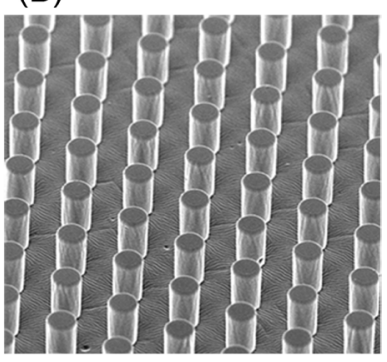

Figure 1. Typical examples of brightfield images (A) ( $\times 40$ objective lens) and scanning electron micrographs (B) [13] of the PDMS micropillar substrate with a hexagonal arrangement. 
PDMS substrate was $\sim 1.6 \mathrm{kPa}$ [13], which was of the same order of magnitude as extracellular matrices such as elastin $(\sim 0.6 \mathrm{MPa}[14])$ surrounding VSMCs in vivo.

\subsection{Cell Culture}

Porcine aortic smooth muscle cell lines (VSMCs; CAP35405, Cell Applications) were used as the test model. VSMCs were cultured in Dulbecco's Modified Eagle's Medium (Invitrogen) supplemented with 10\% fetal bovine serum (JRH Bioscience), penicillin (100 unit $/ \mathrm{ml})$, and streptomycin $(100 \mu \mathrm{g} / \mathrm{ml})($ Sigma) in a $5 \%$ $\mathrm{CO}_{2}$ incubator at $37^{\circ} \mathrm{C}$. The cells were passaged repeatedly at a $1: 4$ split ratio when they reached $\sim 80 \%$ confluence. Cells at passages 6 - 8 were used for all experiments.

\subsection{Measurement of Cell Migration on the Micropillar Substrate}

Both micropillar array substrates and the flat substrates made of the same lot of PDMS were placed in 35-mm glass-bottom culture dishes (No. 0, Matsunami, Osaka, Japan). The surfaces of both substrates were exposed to oxygen plasma ( 5 $\mathrm{mA}, 10 \mathrm{~Pa}$ ) for 2 min using a plasma generator (SEDE-P, Meiwafosis, Tokyo, Japan). All surfaces of both the pillar and flat substrates were coated with fibronectin $(50 \mu \mathrm{g} / \mathrm{ml}$, Sigma) to allow cell adhesion. VSMCs were cultured on these two substrates in DMEM supplemented with $10 \% \mathrm{FBS}$ at $37^{\circ} \mathrm{C}$. The initial cell density was controlled to $\sim 50$ cells $/ \mathrm{mm}^{2}$. Time course images of the cells cultured on the flat and micropillar substrates were obtained for $24 \mathrm{~h}$ at 3-min interval using a microscope imaging system with a compact $\mathrm{CO}_{2}$ incubator (BZ-X700, Keyence, Japan) to analyze cell migration on the substrates. The changes in the number of cells were also measured in the same areas of the substrates during culture; the specimen dishes were set on an inverted microscope, and their XYZ coordinates of the regions of interest ( 24 sets, $800 \mu \mathrm{m} \times 800 \mu \mathrm{m}$ ) were recorded. Then the bright field images of the cells at the same location on the substrates were obtained at every observation period during the culture period.

\subsection{Cell Cycle Analysis Using 5-Ethynyl-2-Deoxyuridine (EdU)}

EdU (5-ethynyl-29-deoxyuridine) is a nucleoside analog of thymidine that is incorporated into DNA only during synthesis, allowing visualization of the newly synthesized DNA (Zeng et al. 2016). This method is a less toxic alternative to BrdU incorporation. VSMCs seeded on the micropillar array substrates and the flat substrates were preincubated at $37^{\circ} \mathrm{C}$ for $24 \mathrm{~h}$ and treated with EdU in a dedicated buffer (Click iT EdU Imaging Kits, Molecular Probes) for $4 \mathrm{~h}$ and $12 \mathrm{~h}$, as per the manufacturer's protocol, to ensure the capture of the majority of proliferating cells. The cells were fixed, permeabilized, blocked for nonspecific protein binding, and stained with Alexa Fluor 488 for 30 min under $\mathrm{Cu}(\mathrm{I})$-catalyzed click reaction conditions, as described by the manufacturer. The cells were washed with PBS and fluorescently stained for their intranuclear DNA, as described in the previous section. 


\subsection{Estimation of the Contractile Protein Expression at the Level of the Single Cell}

Standard methods to quantify intracellular protein expression, such as Western blotting, require large number of specimen cells $\left(>10^{6}\right.$ cells) and it cannot be used for the analysis of protein expression in a single cell level. However, it was unable to obtain a sufficient amount of protein for western blot analysis in this study. Thus, in order to investigate the effects of the mechanical deformation of the nucleus on the smooth muscle contractile protein expression, such as $\alpha$-smooth muscle actin ( $\alpha$-SMA), the fluorescence intensity was measured accurately in a single cell level using the method described previously [15]. Briefly, VSMCs cultured on the micropillar and flat substrates for 3 days were detached with trypsinization and moved to the glass-bottom culture dishes coated with poly-D-lysine. Then, the detached round-shaped cells were fixed immediately with a phosphate buffered saline containing 3.7\% formaldehyde for $5 \mathrm{~min}$, permeabilized with PBS containing $0.5 \%$ Triton X-100 for $5 \mathrm{~min}$, and rinsed with PBS containing $1 \%$ BSA. For $\alpha$-SMA staining, the fixed and permeabilized cells were incubated for $1 \mathrm{~h}$ at $37^{\circ} \mathrm{C}$ with $\alpha$-SMA antibody (clone 1A4 (A2547), Sigma) in PBS containing $1 \%$ bovine serum albumin. After washing, the cells were incubated for $1 \mathrm{~h}$ at room temperature with Alexa Fluor 488-conjugated secondary antibody (Molecular Probes; 1:200). Actin filaments in the cells were also stained fluorescently by incubation with Alexa Fluor 546-conjugated phalloidin (Molecular Probes, USA) at a concentration of $\sim 200 \mathrm{nM}$ for $1 \mathrm{~h}$ at room temperature.

The stained isolated cells were set on the stage of an inverted fluorescent microscope equipped with a confocal system (CSU-X1; Yokogawa, Tokyo, Japan) with a multicolor light emitting diode (LED) fluorescence system (Light Engine Spectra-X; Opto-line, Osaka, Japan) and a digital complementary metal-oxide semiconductor (CMOS) camera (ORCA-Flash4.0 V2; Hamamatsu Photonics, Hamamatsu City, Japan). The fluorescence images slices of their actin filaments and $\alpha$-SMA were obtained in the same cell using an oil-immersion objective (60×, $\mathrm{NA}=1.40)$ and recorded in a personal computer. These image slices were collected in the range of the thickness of the isolated cells $(\sim 25 \mu \mathrm{m})$ at a $0.5-\mu \mathrm{m}$ interval. The reconstructed plane images were obtained with an integration of the slices and their fluorescent intensity was measured as indices of the intracellular expression of actin filaments and $\alpha$-SMA.

\subsection{Statistical Analysis}

Data were expressed as mean \pm SD. Differences were analyzed by the Student's paired and unpaired $t$-test. For cell proliferation, data were assessed using ANOVA with a correction for multiple comparisons, followed by a Steel-Dwass test for multiple comparisons of the means between two groups using the statistical analysis program MEPHAS (in Japanese, http://www.gen-info.osaka-u.ac.jp/testdocs/tomocom/). $P$-values $<0.05$ were considered significant for all analyses. 


\section{Results and Discussion}

Typical fluorescent images of VSMCs cultured on the PDMS micropillars and flat substrates are shown in Figure 2. The cells spread completely between the fibronectin-coated pillars at day 1 , leading to strong deformations of their nuclei (Figure 2(D)). The nuclei in VSMCs were largely deformed and entirely inserted into the grooves between the pillars and they appeared to be "trapped" mechanically on the array of pillars, although they remained in an elongated shape (Figures 2(D)-(F)). Such nuclear deformation did not affect cell viability; the viabilities of VSMCs on the pillar substrate were greater than 98\% [16]. The results demonstrated that the cell nuclei had the ability to deform in response to the micropillared surface. In contrast, on the flat surface, cell nuclei had elliptical shapes with smooth surfaces (Figures 2(A)-(C)).

The migration trajectories of the VSMCs were measured with the captured images (Figure 3), revealing that their migration value was significantly inhibited on the array of pillars. Interestingly, the cell migration had a relatively straight directional trajectory on the micropillar substrates compared to on the flat substrates (Figure $3(B)$ ). The cells on the flat substrates migrated $\sim 13 \mu \mathrm{m} / \mathrm{h}$ on average, and the migration rate was less than half on the micropillar substrates (Figure 3(C)).
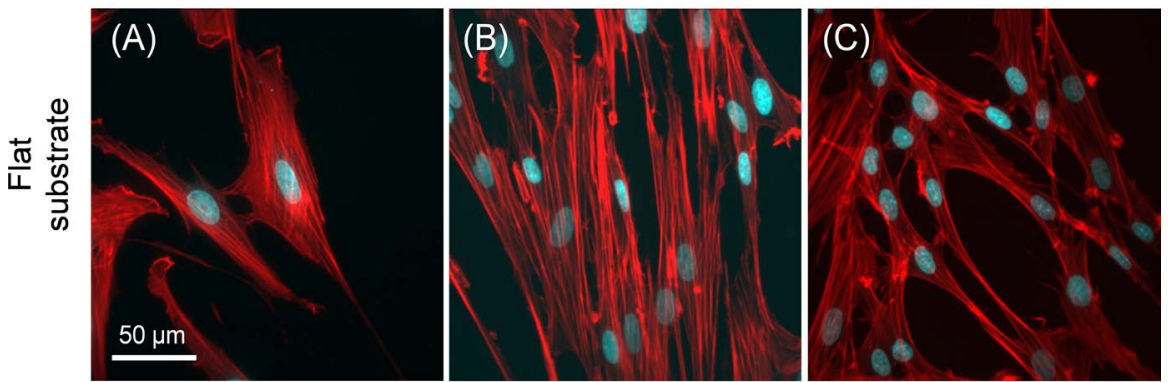

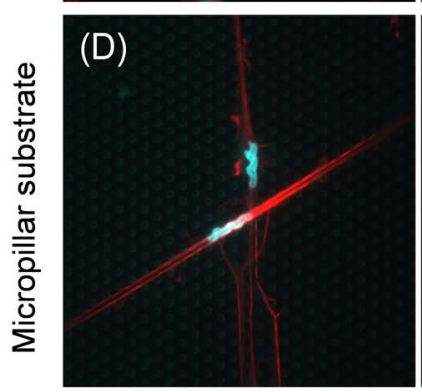

Day 1

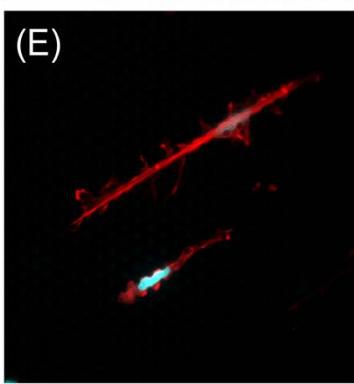

Day 3

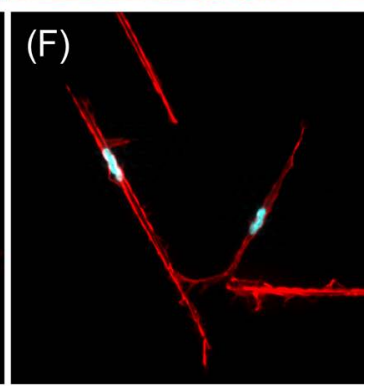

Day 5

Figure 2. Typical examples of fluorescence images of actin filaments (red) and nuclei (cyan) in vascular smooth muscle cells (VSMCs) $(\times 40$ objective lens) The cells were cultured on the polydimethylsiloxane (PDMS) substrates, which have flat surfaces (A)-(C) or the array of micropillars (D)-(F) for 5 days. The changes in the proliferation of SMCs (I) and HeLa cells (J) during culture. The cell proliferation is represented as the ratio of number of cells at a particular day to that at day $0 .{ }^{\star} P<0.05$ vs. the measurement at two days before. Differences in the migration speed of the cells on the flat and micropillar substrates (K). 
(A) Flat substrate

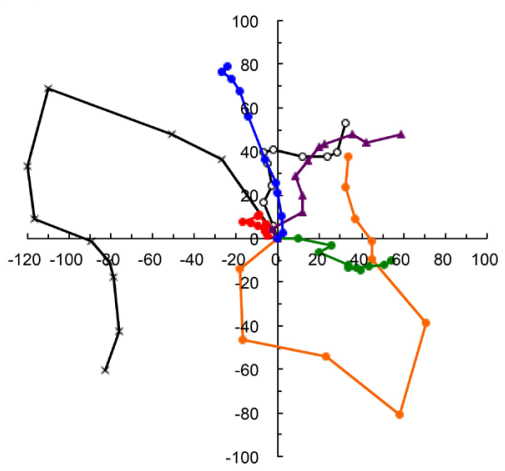

(B) Micropillar substrate

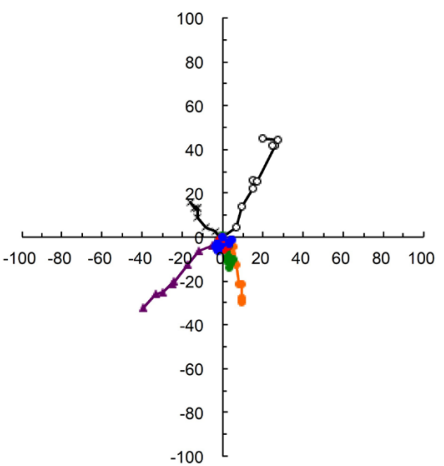

(C)

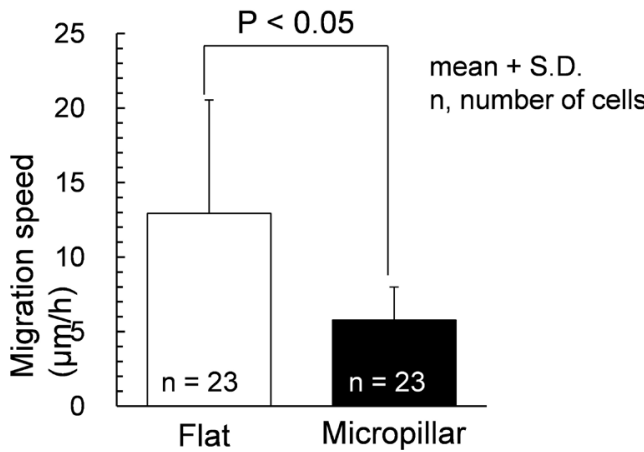

Figure 3. Examples of the migration trajectory of the vascular smooth muscle cells (VSMCs) on the flat substrates (A) and the micropillar substrates (B). The migration trajectory of each cell was represented by the lines in different colars. Differences in the migration speed of the VSMCs on the flat and micropillar substrates (C).

The number of VSMCs on the pillar substrates was mostly unchanged (Figures 2(D)-(F)), although the numbers of cells on the flat substrate increased normally (Figures 2(A)-(C)). A statistical analysis of cell numbers revealed that nuclear trapping with the micropillar substrates significantly inhibited the proliferation of VSMCs (Figure 4). We also assessed the ratio of S-phase cells in the three groups, as detected with EdU assay and found that the DNA synthesis during cell cycle significantly inhibited on the micropillar substrates (Figure 5). Furthermore, a statistical analysis of the contractile protein expression at single cell level using confocal microscopy revealed that expression of both actin filaments and $\alpha$-SMA were significantly reduced in the cell with deformed nuclei cultured on the micropillars (Figure $6, P<0.01$ ). It has generally been suggested that cell-cell contact inhibition of cell proliferation occurs when a cell culture reaches confluence [17] and such proliferation inhibition also promoted expression level of $\alpha$-SMA [18]. However, the both proliferation and contractile protein expression of VSMCs were significantly inhibited in the micropillar substrates even though the cells did not reach the confluent state (compare Figure 2(C) and Figure 2(F)). These results indicate that nuclear mechanical trapping with the micropillars, subsequently inducing substantial deformation of the nuclei, significantly inhibited the migration, proliferation, and contractile differentiation of VSMCs, and led the cells to a "quiescent state". 


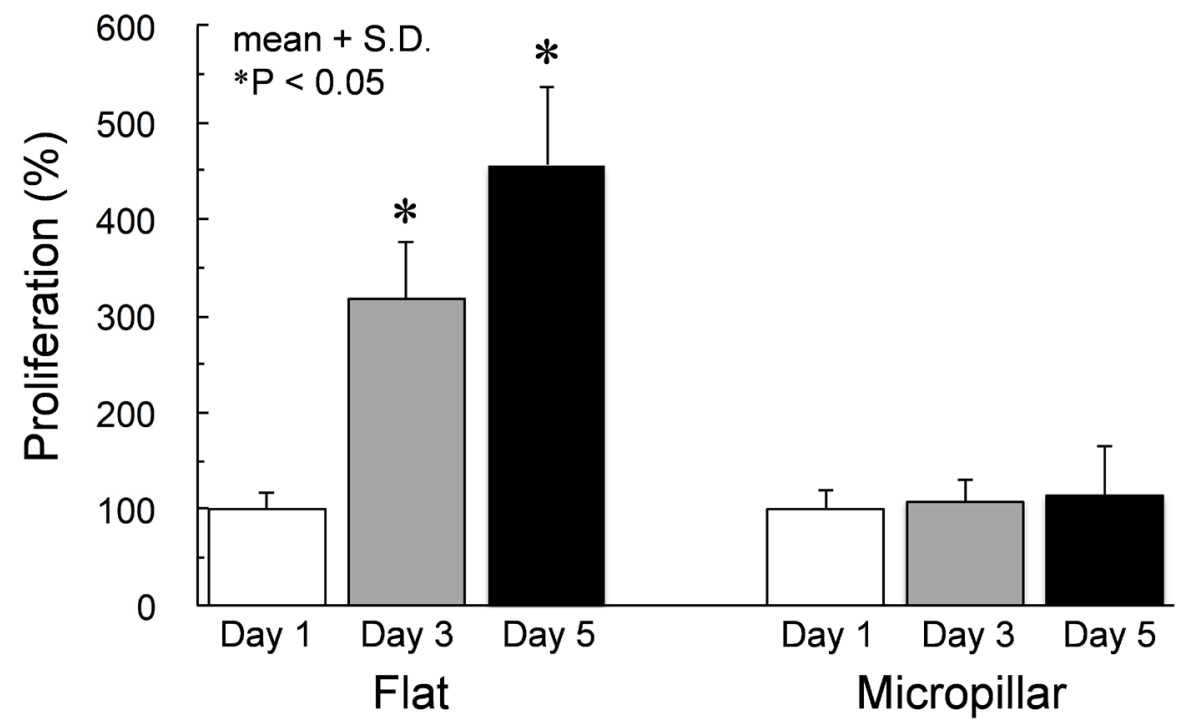

Figure 4. The changes in the proliferation of vascular smooth muscle cells (VSMCs) during culture. The VSMC proliferation is represented as the ratio of number of cells at a particular day to that at day $0 .{ }^{\star} P<0.05$ vs. the measurement at two days before.

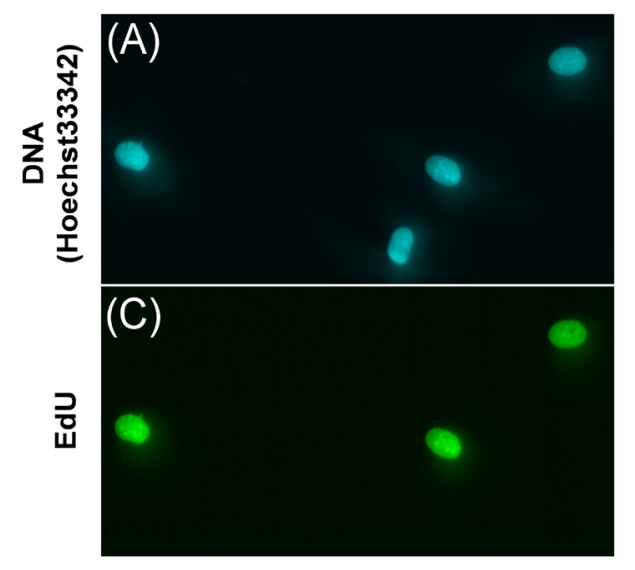

(E)
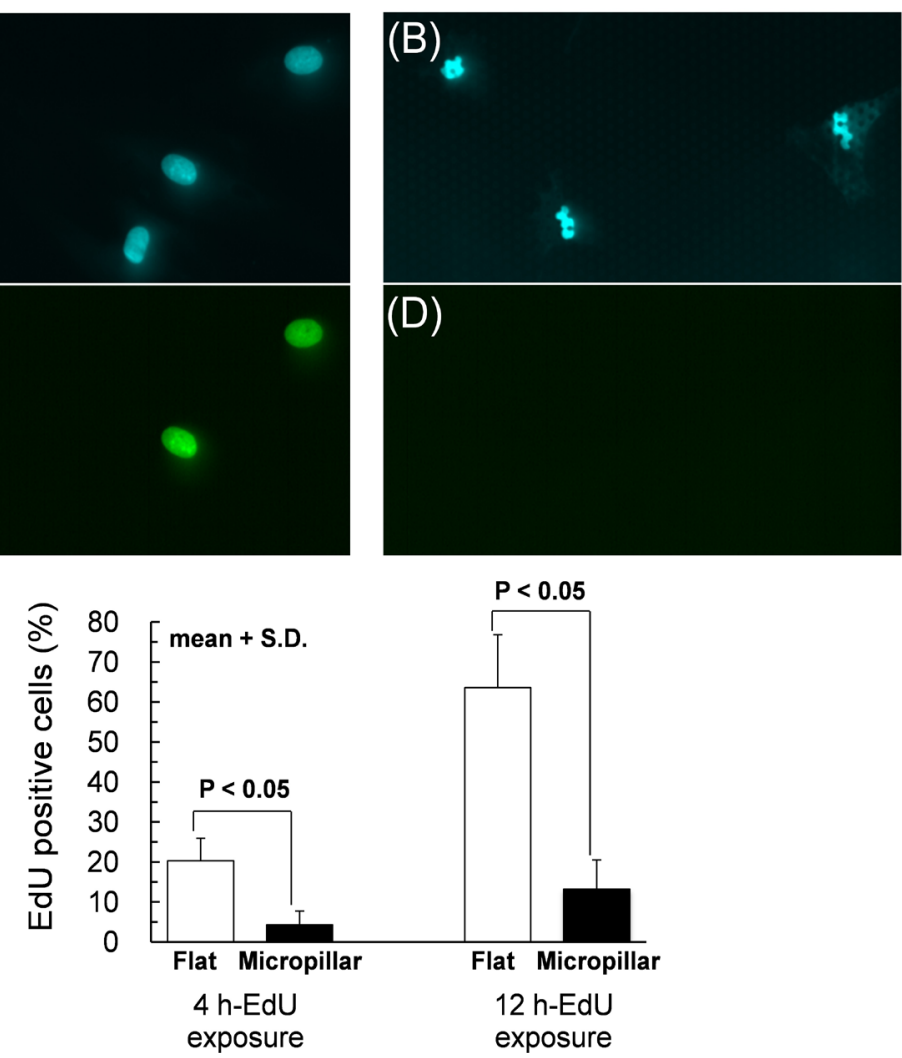

$P<0.05$

Figure 5. Effects of nucleus deformation with a micropillar array on the proliferation of vascular smooth muscle cells (VSMCs), as assessed with EdU assay. Intranuclear DNA was stained with Hoechst 33342 ((A), (B)) and compared with those from EdU-posittive cell nuclei ((C), (D)) in VSMCs on the flat substrates ((A), (C)) and on the micropillar substrates ((B), (D)). Note that EdU-positive cell nucleus was not detected in (D). A significant difference was observed in the ratio of S-phase cells in both 4-hours and 12-hours EdU exposure (E). Over 100 images were analyzed in each group. 

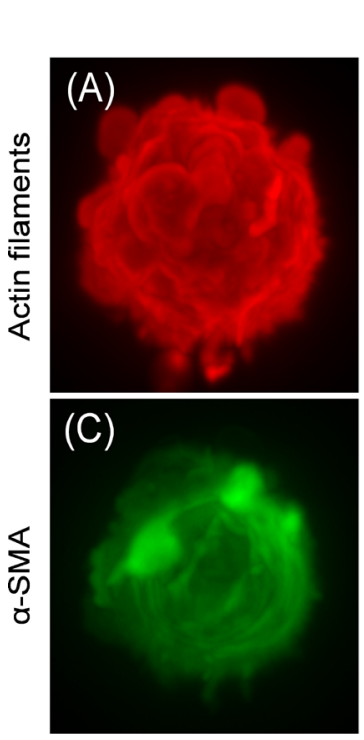

Detached cell from the flat substrate
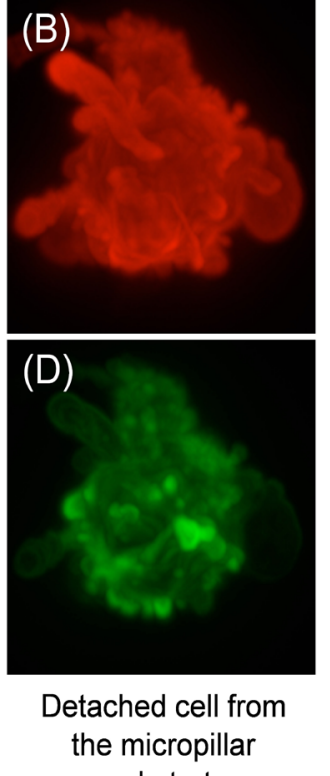

substrate
(E)

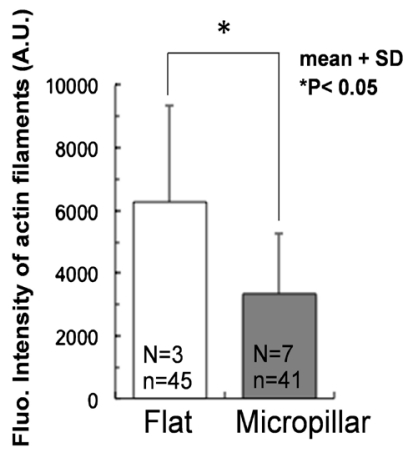

$(\mathrm{F})$

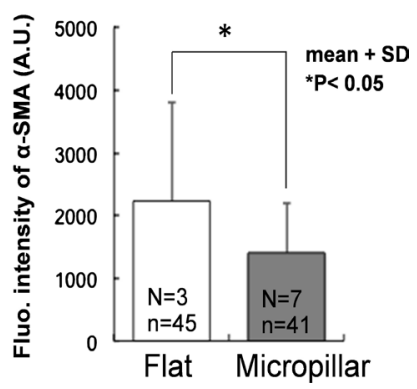

Figure 6. Examples of the reconstructed projection images of actin filaments ((A), (B)) and $\alpha$-SMA ((C), (D)) in vascular smooth muscle cells (VSMCs) detached from the flat ((A), (C)) and micropillar ((B), (D)) substrates, and their average fluorescent intensity $((\mathrm{E}),(\mathrm{F}))$.

\section{Acknowledgements}

This work was supported in part by a Grant-in-Aid from the Ministry of Education, Culture, Sports, Science and Technology, Japan (nos. 17H02077 and 19K22944); the Naito Foundation, Japan; the Takahashi Industrial and Economic Research Foundation, Japan; and AMED-CREST from Japan Agency for Medical Research and Development, AMED (JP19gm0810005 and JP20gm0810005).

\section{Conflicts of Interest}

The author declares no conflicts of interest regarding the publication of this paper.

\section{References}

[1] Terry, L.J., Shows, E.B. and Wente, S.R. (2007) Crossing the Nuclear Envelope: Hierarchical Regulation of Nucleocytoplasmic Transport. Science, 318, 1412-1416. https://doi.org/10.1126/science.1142204

[2] Pemberton, L.F. and Paschal, B.M. (2005) Mechanisms of Receptor-Mediated Nuclear Import and Nuclear Export. Traffic, 6, 187-198.

https://doi.org/10.1111/j.1600-0854.2005.00270.x

[3] Ingber, D.E. (1990) Fibronectin Controls Capillary Endothelial Cell Growth by Modulating Cell Shape. Proceedings of the National Academy of Sciences of the United States of America, 87, 3579-3583. https://doi.org/10.1073/pnas.87.9.3579 
[4] Engler, A.J., Sen, S., Sweeney, H.L. and Discher, D.E. (2006) Matrix Elasticity Directs Stem Cell Lineage Specification. Cell, 126, 677-689. https://doi.org/10.1016/j.cell.2006.06.044

[5] Chen, C.S., Mrksich, M., Huang, S., Whitesides, G.M. and Ingber, D.E. (1997) Geometric Control of Cell Life and Death. Science, 276, 1425-1428. https://doi.org/10.1126/science.276.5317.1425

[6] Smith, P.G., Roy, C., Fisher, S., Huang, Q.Q. and Brozovich, F. (2000) Selected Contribution: Mechanical Strain Increases Force Production and Calcium Sensitivity in Cultured Airway Smooth Muscle Cells. Journal of Applied Physiology, 89, 2092-2098. https://doi.org/10.1152/jappl.2000.89.5.2092

[7] Dahl, K.N., Ribeiro, A.J. and Lammerding, J. (2008) Nuclear Shape, Mechanics, and Mechanotransduction. Circulation Research, 102, 1307-1318.

https://doi.org/10.1161/CIRCRESAHA.108.173989

[8] Lombardi, M.L., Jaalouk, D.E., Shanahan, C.M., Burke, B., Roux, K.J. and Lammerding, J. (2001) The Interaction between Nesprins and Sun Proteins at the Nuclear Envelope Is Critical for Force Transmission between the Nucleus and Cytoskeleton. The Journal of Biological Chemistry, 286, 26743-2653.

https://doi.org/10.1074/jbc.M111.233700

[9] Nagayama, K., Yahiro, Y. and Matsumoto, T. (2011) Stress Fibers Stabilize the Position of Intranuclear DNA through Mechanical Connection with the Nucleus in Vascular Smooth Muscle Cells. FEBS Letters, 585, 3992-3997. https://doi.org/10.1016/j.febslet.2011.11.006

[10] Roca-Cusachs, P., Alcaraz, J., Sunyer, R., Samitier, J., Farré, R. and Navajas, D. (2008) Micropatterning of Single Endothelial Cell Shape Reveals a Tight Coupling between Nuclear Volume in G1 and Proliferation. Biophysical Journal, 94, 4984-4995. https://doi.org/10.1529/biophysj.107.116863

[11] Versaevel, M., Grevesse, T. and Gabriele, S. (2012) Spatial Coordination between Cell and Nuclear Shape within Micropatterned Endothelial Cells. Nature Communications, 3, 671. https://doi.org/10.1038/ncomms1668

[12] Tan, J.L., Tien, J., Pirone, D.M., Gray, D.S., Bhadriraju, K. and Chen, C.S. (2003) Cells Lying on a Bed of Microneedles: An Approach to Isolate Mechanical Force. Proceedings of the National Academy of Sciences of the United States of America, 100, 1484-1489. https://doi.org/10.1073/pnas.0235407100

[13] Nagayama, K., Adachi, A. and Matsumoto, T. (2011) Heterogeneous Response of Traction Force at Focal Adhesions of Vascular Smooth Muscle Cells Subjected to Macroscopic Stretch on a Micropillar Substrate. Journal of Biomechanics, 44, 2699-2705. https://doi.org/10.1016/j.jbiomech.2011.07.023

[14] Fung, Y.C. (1981) Bio-Viscoelastic Solids. In: Biomechanics, Springer, New York, 196-214. https://doi.org/10.1007/978-1-4757-1752-5_7

[15] Nagayama, K., Morishima, N. and Matsumoto, T. (2009) Effects of Three-Dimensional Culture and Cyclic Stretch Stimulation on Expression of Contractile Proteins in Freshly Isolated Rat Aortic Smooth Muscle Cells. Journal of Biomechanical Science and Engineering, 4, 286-297. https://doi.org/10.1299/jbse.4.286

[16] Nagayama, K., Hamaji, Y., Sato, Y. and Matsumoto, T. (2015) Mechanical Trapping of the Nucleus on Micropillared Surfaces Inhibits the Proliferation of Vascular Smooth Muscle Cells But Not Cervical Cancer HeLa Cells. Journal of Biomechanics, 48, 1796-1803. https://doi.org/10.1016/j.jbiomech.2015.05.004

[17] Hneino, M., Bouazza, L., Bricca, G., Li, J.Y. and Langlois, D. (2009) Density-Dependent Shift of Transforming Growth Factor-Beta-1 from Inhibition to Sti- 
mulation of Vascular Smooth Muscle Cell Growth Is Based on Unconventional Regulation of Proliferation, Apoptosis and Contact Inhibition. Journal of Vascular Research, 46, 85-97. https://doi.org/10.1159/000142612

[18] Blank, R.S., Thompson, M.M. and Owens, G.K. (1988) Cell Cycle versus Density Dependence of Smooth Muscle Alpha Actin Expression in Cultured Rat Aortic Smooth Muscle Cells. Journal of Cell Biology, 107, 299-306.

https://doi.org/10.1083/jcb.107.1.299 